\title{
Relations of neuropeptide $Y$ and heme oxygenase-1 expressions with fetal brain injury in rats with intrahepatic cholestasis of pregnancy ${ }^{1}$
}

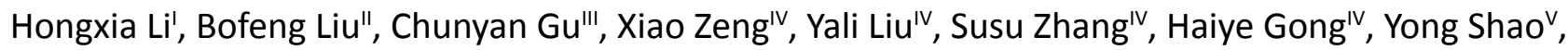
Zhenwei Yaov, Ruifang An ${ }^{\mathrm{VI}}$ (i)

'Doctor, Department of Obstetrics and Gynecology, The First Affiliated Hospital, Xi'an Jiao Tong University; and Department of Gynecology, Affiliated Hospital of Yan'an University, China. Design of the study, acquisition of data, technical procedures, final approval.

"Master, Department of Anatomy, Yan'an University Medical College, China. Acquisition of data, technical procedures, manuscript preparation, final approval.

'"'Bachelor, Department of Gynecology, Affiliated Hospital of Yan'an University, China. Acquisition of data, technical procedures, final approval.

Iv Master, Affiliated Hospital of Yan'an University, China. Acquisition of data, technical procedures, final approval.

${ }^{\vee}$ Master, Department of Obstetrics and Gynecology, The First Affiliated Hospital, Chongqing Medical University, China. Statistical analysis, manuscript preparation, final approval.

V'Doctor, Department of Obstetrics and Gynecology, The First Affiliated Hospital, Xi'an Jiao Tong University, China. Design of the study, manuscript preparation, final approval.

\section{Abstract}

Purpose: To investigate the relations of neuropeptide Y (NPY) and heme oxygenase-1 (HO-1) expressions with fetal brain injury in rats with intrahepatic cholestasis of pregnancy (ICP).

Methods: Sixty rats pregnant for 15 days were randomly divided into experimental and control groups. The ICP model was established in experimental group. On the 21st day, the blood biochemical test, histopathological examination of pregnant rat liver and fetal brain tissues and immunohistochemical analysis of fetal rat brain tissues were performed.

Results: On the 21st day, the alanineaminotransferase, aspartate aminotransferase and total bile acid levels in experimental group were significantly higher than control group $(P<0.01)$. Compared with control group, there was obvious vacuolar degeneration in pregnant rat liver tissue and fetal brain tissue in experimental group. NPY expression in fetal brain tissue was negative in control group and positive in experimental group. HO-1 expression in fetal brain tissue was strongly positive in control group and positive in experimental group. There was significant difference of immunohistochemical staining optical density between two groups $(\mathrm{P}<0.01)$.

Conclusion: In fetal brain of ICP rats, the NPY expression is increased, and the HO-1 expression is decreased, which may be related to the fetal brain injury.

Key words: Cholestasis, Intrahepatic. Neuropeptide Y. Heme Oxygenase-1. Brain. Rats. 


\section{Introduction}

Intrahepatic cholestasis of pregnancy (ICP) is a special complication of pregnancy, which occurs in the middle and late stage of pregnancy. The main clinical manifestations of ICP include pruritus, jaundice, elevated serum bile acid level and slight elevation of hepatic transaminase ${ }^{1}$. The prognosis of pregnant women with ICP is good, but the fetuses are prone to premature delivery, distress, asphyxia, central nervous system injury, and even intrauterine fetal death, which lead to the significantly increased perinatal mortality rate and caesarean section rate ${ }^{2}$. At present, the etiology and pathogenesis of ICP are not very clear. They are mainly related to estrogen factors, family genetic factors, and environmental factors ${ }^{3,4}$. With the development of neuroendocrine immunology, some scholars have paid more and more attention to the role of neuroendocrine immunity in the process of ICP. Neuropeptide $Y$ (NPY) is a polypeptide composed of 36 amino acids. It acts as the neurotransmitter, and plays a role in nerve regulation and nerve secretion ${ }^{5}$. NPY is widely distributed in the tissues of body, especially in the nervous system. It is related to the brain injury, cerebral apoplexy and neonatal hypoxic-ischemic encephalopathy (HIE) ${ }^{6}$. Heme oxygenase (HO) is a family of microsomal enzymes with high conservatism in evolution. It has important function in regulation of iron ion stability and antioxidant defense ${ }^{7}$. The metabolite of HO, carbon monoxide, acts as a gas neurotransmitter and plays an important role in vascular dilation ${ }^{8}$. There are three isozymes of $\mathrm{HO}$, including HO-1, HO-2 and HO-3. HO-1 is an inducible type, and is widely distributed in the spleen, liver, bone marrow and other tissues. The current studies are focuses on HO- $1^{9,10}$. It is found that, HO-1 is related to the hypertensive disorder complicating pregnancy and intrauterine growth retardation ${ }^{11}$. Until now the relations of NPY and HO-1 with ICP is seldom reported. This study established a pregnant rat model of ICP, and investigated the relations of NPY and HO-1 expressions with fetal brain injury in rats with ICP. The objective was to provide one more theoretical basis for further studying the etiology of ICP and its clinical treatment.

\section{Methods}

This study was carried out in strict accordance with the recommendations in the Guide for the Care and Use of Laboratory Animals of the National Institutes of Health. The animal use protocol has been reviewed and approved by the Institutional Animal Care and Use Committee (IACUC) of The First Affiliated Hospital of Xi'an Jiao Tong University.

\section{Animals and grouping}

A total of 60 female Sprague-Dawley rats (5-6 months of age; $200-250 \mathrm{~g}$; provided by Laboratory Animal Centre, The Third Minitary Medical University, Chongqing, China) were raised in a barrier system (avoiding strong light and noise; room temperature; relative humidity $60 \%-70 \% ; 12 / 12$-h day-night cycle; free to feed and water). In the estrus, the female rats were raised with male SD rats (5-6 months of age; 200-250 g; provided by Laboratory Animal Centre, The Third Minitary Medical University, Chongqing, China) in one cage, with male/female ratio of $4: 1$. The shedding of the vaginal plug was observed every day. The day of vaginal plug shedding was defined as the first day of pregnancy. The rats were raised to the 15th day of pregnancy. Then the pregnant rats were randomly divided into experimental group and control group, 30 rats in each group. 


\section{Establishment of ICP model}

ICP model of rats was established according to the reported methods ${ }^{12,13}$. In the experimental group, $75 \mathrm{mg} / \mathrm{kg}$ progesterone (Sigma-Aldrich Corp., MO, US) and $1.25 \mathrm{mg} /$ kg 17- $\alpha$-ethinyl estradiol (Sigma-Aldrich Corp., MO, US) were injected subcutaneously at the medial hind limb, once every day from the 15th day of pregnancy. In control group, the $2.5 \mathrm{ml} /$ $\mathrm{kg}$ refined oil (Chongqing Chemical Reagent Co., Ltd., Chongqing, China) was injected subcutaneously at the medial limb at the time the same with experimental group.

\section{Determination of blood biochemical indexes}

At the 21st day of pregnancy, $1 \mathrm{ml}$ of blood was taken from the orbital vein, and the blood biochemical indexes including alanineaminotransferase (ALT), aspartate aminotransferase (AST) and total bile acid (TBA) were determined using TBA-2000FR automatic biochemical analyzer (Toshiba Medical Systems China Co., Ltd., Beijing, China).

\section{Histopathological examination}

Thirty pregnancy rats were sacrificed by twisting neck. The pregnant rat liver and fetal brain tissues were taken and fixed immediately using $4 \%$ paraformaldehyde. After paraffin embedding and hematoxylin-eosin staining, the histopathological changes were observed under DVM6 optical microscope (Leica Science Lab, Leica Camera AG Berlin, Germany).

\section{Immunohistochemical staining}

The paraffin sections of fetal rat brain tissues were prepared. $2 \mu \mathrm{m}$ paraffin sections were deparaffinized, and then washed with distilled water. After soaking in PBS for 5 min, they were incubated with $3 \% \mathrm{H}_{2} \mathrm{O}_{2}$ at room temperature for $15 \mathrm{~min}$. After washing with
PBS, the appropriate proportion of primary antibodies (rabbit anti-rat NPY, rabbit anti-rat HO-1; Wuhan Boster Bioengineering Co., Ltd., Wuhan, China) were added for incubation at $37^{\circ} \mathrm{C}$ for $1 \mathrm{~h}$, followed by washing with PBS. Then the biotinylated secondary antibody (HRPlabeled goat anti-rabbit IgG, Beijing Zhongshan Golden Bridge Biotechnology Co., Ltd., Beijing, China) was added, followed by incubation at $37^{\circ} \mathrm{C}$ for $30 \mathrm{~min}$. After washing with PBS, the DAB (Wuhan Boster Bioengineering Co., Ltd., Wuhan, China) staining was performed, followed by washing with water, restaining, dehydration and mounting. The semiquantitative analysis was performed with 801 image analysis system (Jiangsu Jieda Technology Development Co., Ltd., Nanjing, China). Two sections were randomly selected from each group, and 5 visual fields of each section were selected. The staining intensity was presented using average optical density (OD).

\section{Statistical analysis}

All statistical analysis was carried out using SPSS22.0 software (SPSS Inc., Chicago, IL, USA). The measurement data were presented as mean $\pm S D$, and were compared using $t$ test. $\mathrm{P}<0.05$ was considered as statistically significant.

\section{- Results}

Biochemical indexes of pregnant rats in two groups

As shown in Table 1, at the 21st day of pregnancy, the levels of ALT, AST and TBA in control group were 42.91 $\pm 9.66 \mathrm{IU} / \mathrm{L}$, 49.11 $\pm 11.47 \mathrm{IU} / \mathrm{L}$ and $24.15 \pm 3.32 \mu \mathrm{mol} / \mathrm{L}$, respectively, and those in experimental group 
were $228.95 \pm 9.02 \mathrm{IU} / \mathrm{L}, 432.58 \pm 21.12 \mathrm{IU} / \mathrm{L}$ and $78.37 \pm 6.18 \mu \mathrm{mol} / \mathrm{L}$, respectively. There was significant difference of each index between two groups $(P<0.01)$. This indicated that, the ICP model of rats was successfully established.

Table 1 - Biochemical indicators after inducing model in two group.

\begin{tabular}{lllll}
\hline Group & $\mathbf{n}$ & ALT $(I U / L)$ & AST $(I U / L)$ & TBA $(\mu \mathrm{mol} / \mathrm{L})$ \\
\hline Control & 30 & $42.91 \pm 9.66$ & $49.11 \pm 11.47$ & $24.15 \pm 3.32$ \\
Experimental & 30 & $228.95 \pm 9.02^{*}$ & $432.58 \pm 21.12^{*}$ & $78.37 \pm 6.18^{*}$ \\
\hline
\end{tabular}

${ }^{*} \mathrm{P}<0.01$ compared with control group. ALT, alanineaminotransferase; AST, aspartate aminotransferase; TBA, total bile acid.

Histopathological results of liver tissue in pregnant rats

In control group, there was no obvious morphological change in liver tissue of pregnant rats. In the experimental group, partial liver cells presented granular degeneration and vacuolar degeneration. There were bile plugs in partial capillary bile ducts. The structure of hepatic lobules was normal (Fig. 1).

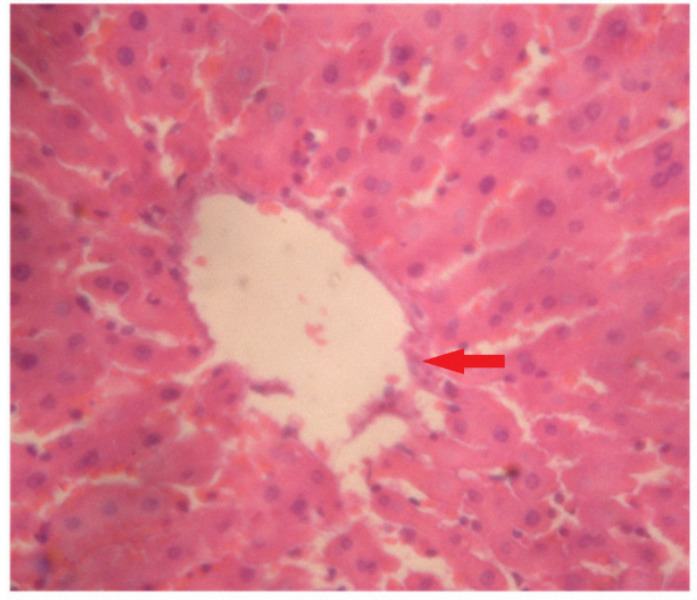

Control group

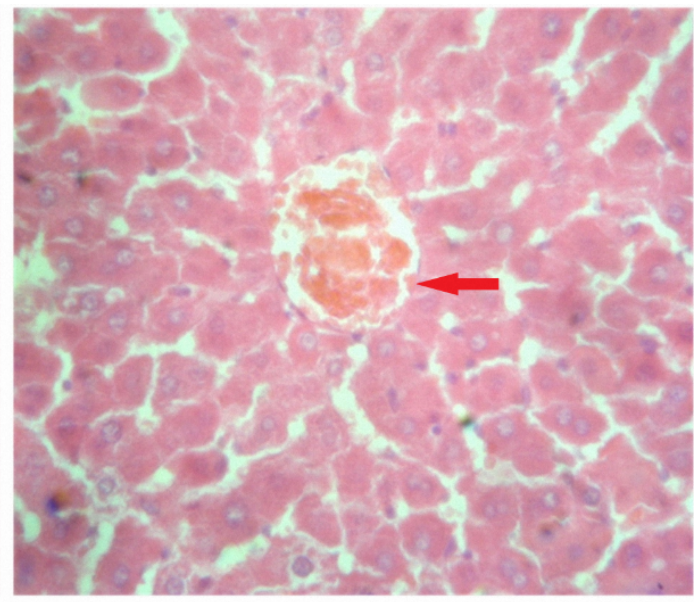

Experimental group

Figure 1 - Histopathological results of liver tissue in pregnant rats in control group and experimental group (hematoxylin-eosin staining, $\times 200$ ). Control group: there was no obvious morphological change in liver tissue of pregnant rats. Experimental group: the partial liver cells presented granular degeneration and vacuolar degeneration.

Histopathological results of brain tissue in fetal rats

In control group, no edema was observed in brain tissue of fetal rats. The cell and intercellular structure were normal. In experimental group, the brain tissue was loose, and the vacuolar degeneration was obvious. The parts of the cells were lysised, and even disappeared (Fig. 2). 


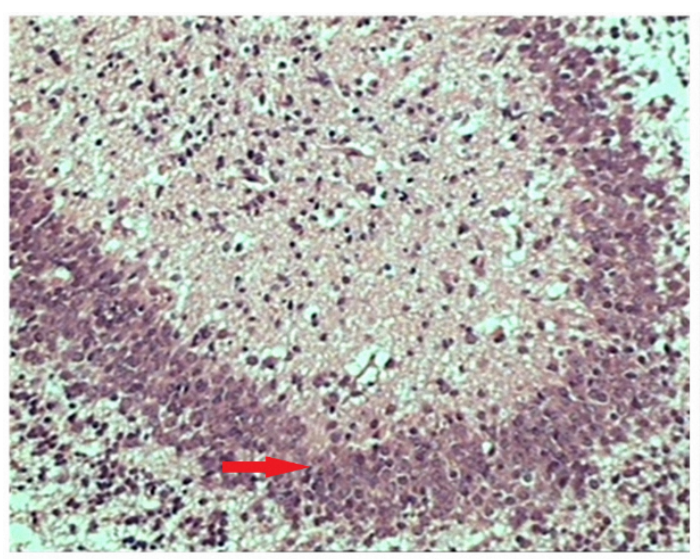

Control group

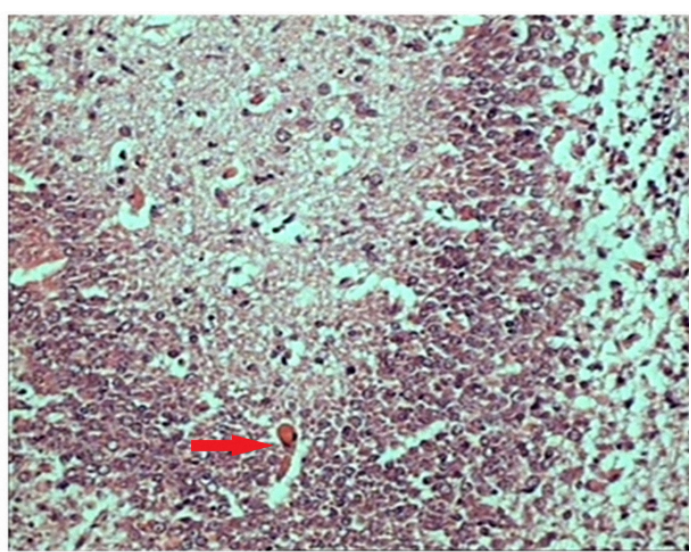

Experimental group

Figure 2 - Histopathological results of fetal brain tissue in control group and experimental group (hematoxylineosin staining, $\times 200$ ). Control group: no edema was observed in brain tissue of fetal rats. The cell and intercellular structure were normal. Experimental group: the brain tissue was loose, and the vacuolar degeneration was obvious. Parts of the cells were lysised, and even disappeared.

Immunohistochemical results of NPY in fetal brain tissue

In control group, there were partial of cell membrane coloration in the hippocampus of fetal brain tissues, without positive NPY-immunoreactive substance. In the experimental group, the positive NPY immunoreactive neurons were mainly located in the hippocampus, which presented brown. The positive immunoreactive granules were mainly distributed in the cytoplasm and surface of the cell membrane (Fig. 3).

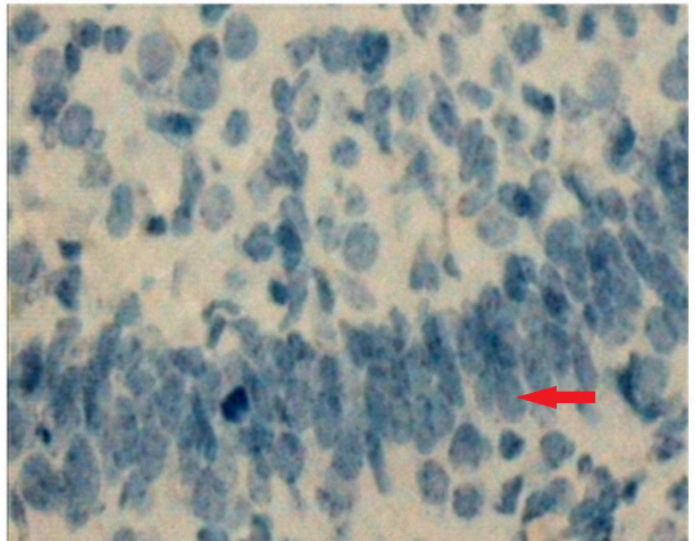

Control group

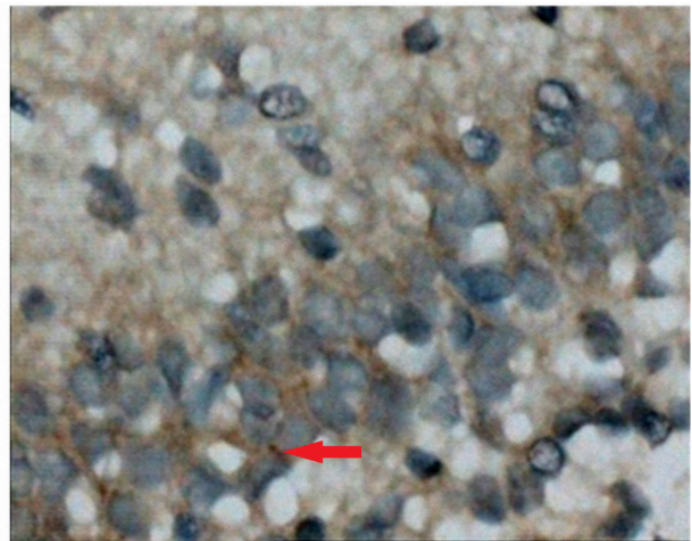

Experimental group

Figure 3 - Negative neuropeptide $Y$ expression in fetal brain tissue in control group and positive neuropeptide $Y$ expression in fetal brain tissue in experimental group $(\times 400)$. Control group: there were partial of cell membrane coloration in the hippocampus of fetal brain tissues, without positive neuropeptide $Y$-immunoreactive substance. Experimental group: the positive neuropeptide $Y$-immunoreactive neurons were mainly located in the hippocampus, which presented brown. The positive immunoreactive granules were mainly distributed in the cytoplasm and surface of the cell membrane. 
Immunohistochemical results of $\mathrm{HO}-1$ in fetal brain tissue

In control group, the HO-1 immunepositive cells in hippocampal CAl region of fetal brain were stained brown, which were mainly distributed in the cytoplasm and cell membrane surface, presenting strongly positive. In the experimental group, there were less immunepositive cells in hippocampal CAl region, which were stained yellow, presenting positive (Fig. 4).

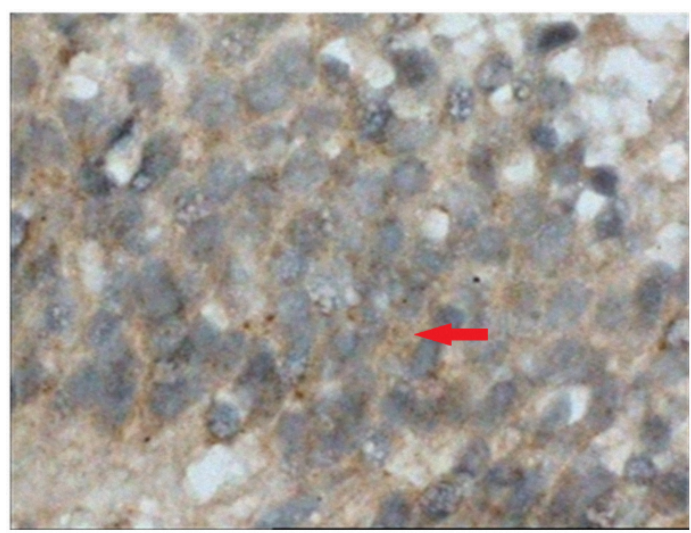

Control group

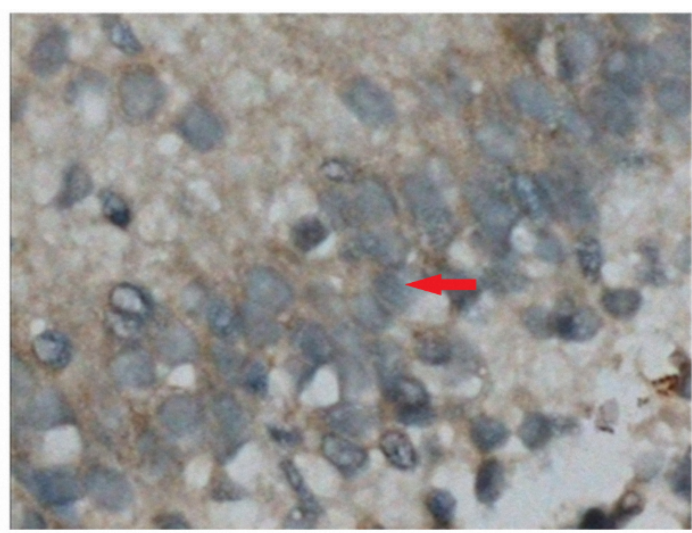

Experimental group

Figure 4 - Strongly positive heme oxygenase-1 expression in fetal brain tissue in control group and positive heme oxygenase- 1 expression in fetal brain tissue in experimental group ( $\times 400)$. Control group: the heme oxygenase-1 immune-positive cells in hippocampal CAl region of fetal brain were stained brown, which were mainly distributed in the cytoplasm and cell membrane surface, presenting strongly positive. Experimental group: there were less heme oxygenase- 1 immune-positive cells in hippocampal CAl region, which were stained yellow, presenting positive.

Image analysis results of immunohistochemical staining

Results of image analysis showed that, the OD value of NPY immunohistochemical staining in experimental group was $1.08425 \pm 0.0179$, which was significantly higher than $0.6324 \pm 0.0246$ in control group $(\mathrm{P}<0.01)$. The OD value of HO-1 immunohistochemical staining in experimental group was $0.8876 \pm 0.0208$, which was significantly higher than $1.638 \pm 0.0271$ in control group $(P<0.01)$ (Table 2).

Table 2 - Image analysis results of immunohistochemical staining in two groups (OD).

\begin{tabular}{llll} 
Group & $\mathbf{n}$ & NPY & HO-1 \\
\hline Control & 30 & $0.6324 \pm 0.0246$ & $1.638 \pm 0.0271$ \\
Experimental & 30 & $1.08425 \pm 0.0179^{*}$ & $0.8876 \pm 0.0208^{*}$ \\
\hline
\end{tabular}

${ }^{*} \mathrm{P}<0.01$ compared with control group. OD, optical density; NPY, neuropeptide Y; HO-1, heme oxygenase-1. Control group. Experimental group. 


\section{- Discussion}

It is reported that, the liver biochemical and pathological changes of pregnant rats after treatment with estrogen are similar to those of human with ICP, and this can be used as an animal model for studying human ICP. However, it is found that the ICP model established by this method shows slight necrosis in the liver under light microscope, which is not consistent with the fact that there is no spotty necrosis change in human $\mathrm{ICP}^{14}$. This suggests that the establishment method of ICP model using estrogen alone is not perfect. Valleio et al. ${ }^{15}$ find that, pregnant women with oral progesterone present the ICP symptoms including itching. Therefore, in some scholars' researches, estrogen and progesterone are combined to establish the ICP animal model, and the liver biochemical and pathological changes of animal are very similar to those of human ICP ${ }^{16}$. In this study, estrogen and progestogen were combined to establish the ICP model of pregnant rats. The blood biochemical test showed that, at the 21st day of pregnancy, the levels of ALT, AST and TBA in the experimental group were significantly higher than those in the control group, respectively $(P<0.01)$. In addition, the liver histological examination showed that, in control group, there was no obvious morphological change in liver. In the experimental group, partial liver cells presented granular degeneration and vacuolar degeneration. There were bile plugs in partial capillary bile ducts. The structure of hepatic lobules was normal. This indicates that, the ICP model of pregnant rats has been successfully established.

Animal clinical experiments have found that, NPY is associated with brain damage, stroke, and neonatal HIE ${ }^{17-19}$. HIE refers to the brain injury caused by reduced oxygen supply reduction and/or reduced blood supply secondary to late pregnancy, delivery or neonatal period. It is the main form of perinatal brain injury, and is the main cause of acute death ${ }^{19}$. Previous study ${ }^{20}$ finds that, in HIE patients the plasma NPY level is increased significantly. The higher the HIE level is, the higher the plasma NPY level is. The NPY level in the recovery phase is decreased significantly. This suggests that, the plasma NPY is involved in the pathophysiological process of HIE. In neonatal asphyxia, the plasma NPY is significantly increased, resulting in the increase of cerebral vascular resistance and the decrease of cerebral blood flow. This becomes one of the causes of $\mathrm{HIE}^{6}$. NPY can inhibit the vasodilation effect of blood vessels on adenosine, acetylcholine and other vasodilator substances. This causes the accommodative disorder of cerebral vasodilatation and contraction function, leading to further contraction of cerebral blood vessels and reduction of cerebral blood flow ${ }^{21}$. In addition, NPY can promote the hydrolysis of phosphatidylinositol on the cell membrane, producing two diacylglycerol and inositol triphosphate (IP3). As a second messenger, IP3 can promote the opening of $\mathrm{Ca}^{2+}$ channels in the endoplasmic reticulum and increase the intracellular $\mathrm{Ca}^{2+}$ concentration, thus aggravating hypoxic ischemic brain damage ${ }^{22}$. The results of this study showed that, NPY was mainly distributed in the hippocampus of ICP fetal rats. The expression of NPY in the experimental group was increased significantly, compared with control group. This suggests that, NPY may be related to fetal distress in ICP.

Recent study has shown that, HO-1 has the anti-inflammatory, anti-apoptotic and anti-proliferative effects, and plays a cytoprotective role in related diseases such as arteriosclerosis, cerebral ischemia and 
organ transplant rejection. Therefore, the biological activity of $\mathrm{HO}-1$ and its protective effect in many clinical diseases have attracted the attention of many scholars. It is found that, the antioxidant role of HO-1 has the best expression threshold ${ }^{23}$. The moderate expression $\mathrm{HO}-1$ can reduce the cell death, protein oxidation and lipid peroxidation, thus promoting the cell proliferation. The over expression of HO-1 may damage the integrity of cells. The low expression of HO-1 leads to the decreased protective effect, thus the cells are susceptible to the invasion of harmful factors $^{24}$. In this study, the expression of HO-1 in the brain tissue of fetal rats was detected. Results showed that, the immune staining of HO-1 in the hippocampus was obvious in the control group, while it was weakened the experimental group. Because the lesion degree of ICP is different, the expression of HO-1 is different. The more severe the lesion is, the weaker the HO-1 expression is, indicating its weaker protective effect. The study in which the transgenic mice are used as experiment materials proves that, the transgenic mice with over expression of HO-1 present significantly reduced infarction focus in brain ischemic injury and permanent focal cerebral ischemia injury. This indicates that, the expression of $\mathrm{HO}-1$ in the ischemia and hypoxia stress state is related to the self protection mechanism of body ${ }^{9-10}$. Therefore, it is speculated that, the abnormal expression of HO-1 is one of the causes of poor prognosis of fetus in ICP.

\section{Conclusions}

In fetal brain of ICP rats, the NPY expression is increased, and the HO-1 expression is decreased, which may be related to the fetal brain injury. This study has provided one more theoretical basis for further studying the etiology of ICP and its clinical treatment. However, whether there are other mechanisms of ICP should be further studied.

\section{References}

1. Dixon PH, Williamson C. The pathophysiology of intrahepatic cholestasis of pregnancy. Clin Res Hepatol Gastroenterol. 2016;40:141-53. doi: 10.1016/j.clinre.2015.12.008.

2. Brouwers L, Koster MP, Page-Christiaens GC, Kemperman H, Boon J, Evers IM, Bogte A, Oudijk MA. Intrahepatic cholestasis of pregnancy: maternal and fetal outcomes associated with elevated bile acid levels. Am J Obstet Gynecol. 2015;212:100.e1-7. doi: 10.1016/j.ajog.2014.07.026.

3. Pauli-Magnus C, Meier PJ, Stieger B. Genetic determinants of drug-induced cholestasis and intrahepatic cholestasis of pregnancy. Semin Liver Dis. 2010;30:147-59. doi: 10.1055/s-0030-1253224.

4. Pařízek $A$, Dušková $M$, Vítek $L$, Šrámková $M$, Hill $M$, Adamcová K, Šimják $P$, Černý A, Kordová $Z$, Vráblíková $H$, Boudová $B$, Koucký M, Malíčková K, Stárka L. The role of steroid hormones in the development of intrahepatic cholestasis of pregnancy. Physiol Res. 2015;64:S203-9. PMID: 26680481.

5. Protas L, Qu J, Robinson RB. Neuropeptide $y$ : neurotransmitter or trophic factor in the heart? News Physiol Sci. 2003;18:181-5. PMID: 14500795.

6. Liu J, Zhao J, Di YF, Guo XX, Zhai GR, Huang $\mathrm{XH}$. The dynamic changes of plasma neuropeptide $y$ and neurotensin and their role in regulating cerebral hemodynamics in neonatal hypoxic-ischemic encephalopathy. Am J Perinatol. 2007;24:435-40. doi: 10.1055/s-2007-985047.

7. Ryter SW, Tyrrell RM. The heme synthesis and degradation pathways: role in oxidant sensitivity. Heme oxygenase has both pro- and antioxidant properties. Free Radic Biol Med. 2000;28:289-309. PMID: 11281297.

8. Němeček $D$, Dvořáková $M$, Sedmíková $M$. 
Heme oxygenase/carbon monoxide in the female reproductive system: an overlooked signalling pathway. Int J Biochem Mol Biol. 2017;8:1-12. PMID: 28123837.

9. Adamiak M, Moore JB 4th, Zhao J, Abdelbaset-Ismail A, Grubczak K, Rzeszotek S, Wysoczynski M, Ratajczak Mz. Downregulation of heme oxygenase 1 (HO1) activity in hematopoietic cells enhances their engraftment after transplantation. Cell Transplant. 2016;25:1265-76. doi: 10.3727/096368915X688957.

10. Askenazi DJ, Halloran B, Patil N, Keeling S, Saeidi B, Koralkar R, Ambalavanan N. Genetic polymorphisms of heme-oxygenase 1 (HO-1) may impact on acute kidney injury, bronchopulmonary dysplasia, and mortality in premature infants. Pediatr Res. 2015;77:793-8. doi: $10.1038 /$ pr.2015.44.

11.Kaartokallio T, Klemetti MM, Timonen A, Uotila J, Heinonen S, Kajantie E, Kere J, Kivinen K, Pouta A, Lakkisto P, Laivuori H. Microsatellite polymorphism in the heme oxygenase-1 promoter is associated with nonsevere and late-onset preeclampsia. Hypertension. 2014;64:172-7. doi: 10.1161/ hypertensionaha.114.03337.

12.Yu L, Ding $Y$, Huang $T$, Huang $X$. Effect of bile acid on fetal lung in rat model of intrahepatic cholestasis of pregnancy. Int J Endocrinol. 2014;2014:308274. doi: $10.1155 / 2014 / 308274$.

13.Chen Z, Hu L, Lu M, Shen Z. Resveratrol reduces matrix metalloproteinases and alleviates intrahepatic cholestasis of pregnancy in rats. Can J Physiol Pharmacol. 2016;94:402-7. doi: 10.1139/cjpp-20150454.

14.Wang C, Chen X, Zhou SF, Li X. Impaired fetal adrenal function in intrahepatic cholestasis of pregnancy. Med Sci Monit. 2011;17:CR265-71. doi: 10.12659/ MSM.881766.

15.Vallejo M, Briz O, Serrano MA, Monte MJ, Marin JJ. Potential role of trans-inhibition of the bile salt export pump by progesterone metabolites in the etiopathogenesis of intrahepatic cheolestasis of pregnancy. J Hepatol. 2006;44:1150-7. doi: 10.1016/j. jhep.2005.09.017.
16.Zhang L, Liu J, Estrogen and progesterone induced intrahepatic cholestasis of pregnancy study. Chin J Obstet Gynecol. 2012;45:283-6.

17. Hodges GJ, Jackson DN, Mattar L, Johnson JM, Shoemaker JK. Neuropeptide $Y$ and neurovascular control in skeletal muscle and skin. Am J Physiol Regul Integr Comp Physiol. 2009;297:R546-55. doi: 10.1152/ ajpregu.00157.2009.

18.Donoso MV, Miranda R, Irarrázaval MJ, Huidobro-Toro JP. Neuropeptide Y is released from human mammary and radial vascular biopsies and is a functional modulator of sympathetic cotransmission. J Vasc Res. 2004;41:387-99. doi: 10.1159/000080900.

19. Chen SH, Cheung RT. Intracerebroventricular injection of a neuropeptide $\mathrm{Y}-\mathrm{Y} 1$ receptor agonist increases while BIBP3226, a Y1 antagonist, reduces the infarct volume following transient middle cerebral artery occlusion in rats. Neuroscience. 2003;116:119-26. PMID: 12535945.

20.Colín-González AL, Orozco-Ibarra M, ChánezCárdenas ME, Rangel-López E, Santamaría A, Pedraza-Chaverri J, Barrera-Oviedo D, Maldonado PD. Heme oxygenase-1 (HO1) upregulation delays morphological and oxidative damage induced in an excitotoxic/ pro-oxidant model in the rat striatum. Neuroscience. 2013;231:91-101. doi: 10.1016/j.neuroscience.2012.11.031.

21.Järvisalo MJ, Jartti L, Karvonen MK, Pesonen U, Koulu M, Marniemi J, Hammar N, Kaprio J, Paakkunainen U, Simell O, Raitakari OT, Rönnemaa T. Enhanced endotheliumdependent vasodilation in subjects with Proline7 substitution in the signal peptide of neuropeptide Y. Atherosclerosis. 2003;167:319-26. PMID: 12818415.

22.Drakulich DA, Walls AM, Toews ML, Hexum TD. Neuropeptide $Y$ receptor-mediated sensitization of ATP-stimulated inositol phosphate formation. J Pharmacol Exp Ther. 2003;307:559-65. doi: 10.1124/ jpet.103.053082.

23. Wong RJ, Zhao H, Stevenson DK. A deficiency in haem oxygenase-1 induces foetal growth restriction by placental vasculature defects. Acta Paediatr. 2012;101:827-34. doi: 10.1111/j.1651-2227.2012.02729.x. 
24.Ewing JF, Maines MD. Regulation and expression of heme oxygenase enzymes in aged-rat brain: age related depression in $\mathrm{HO}-1$ and $\mathrm{HO}-2$ expression and altered stress-response. J Neural Transm (Vienna). 2006;113:439-544.

\section{Correspondence:}

\section{Ruifang An}

Department of Obstetrics and Gynecology,

The First Affiliated Hospital

Xi'an Jiao Tong University

277 Yanta West Road, Xi'an 710000 China

Phone: +86-29-85323338

anrunfang@yeah.net

Received: Dec 06, 2018

Review: Feb 10, 2019

Accepted: Mar 03, 2019
Conflict of interest: none

Financial sources: Education Department of Shaanxi Provincial Government (No. 2013JK0782), Yan'an Science and Technology People-benefit Project (No. 2017HM-02-02), and Research Project of Postgraduate Education and Teaching Reform Project in Yan'an University (No. YDYJG2017014)

${ }^{1}$ Research performed at Laboratory, the First Affiliated Hospital, Xi'an Jiao Tong University, Xi'an, China.

This is an Open Access article distributed under the terms of the Creative Commons Attribution License, which permits unrestricted use, (cc) BY distribution, and reproduction in any medium, provided the original work is properly cited. 